\title{
EDITORIAL
}

\section{HPV vaccination in boys: Determining the clinical relevance of this strategy}

\author{
HARALD ZUR HAUSEN $^{1}$, IOANNIS N. MAMMAS ${ }^{2}$ and DEMETRIOS A. SPANDIDOS ${ }^{2}$ \\ ${ }^{1}$ German Cancer Research Centre (DKFZ), Heidelberg 69120, Germany; ${ }^{2}$ Department of Clinical Virology, \\ School of Medicine, University of Crete, Heraklion 71003, Greece
}

Received July 18, 2017; Accepted August 22, 2017

DOI: $10.3892 /$ etm.2017.5005

Human papillomavirus (HPV) infection is an epitheliotropic viral infection (1) - described in the Hippocratic Corpus (2) -, which affects both adults and children (3). Almost 40 years after the first experiments, which investigated the link between HPV and cervical cancer (1), the implementation of vaccination against HPV into paediatric clinical practice represents one of the most significant steps towards the reduction of HPV-associated morbidity and mortality. The World Health Organisation (WHO) has recommended the establishment of HPV vaccination among girls (4), while the cost-effectiveness of HPV vaccination of girls exists even in low- and middle-income countries $(5,6)$. To date, several studies have evaluated the health impact on the population of including males to HPV vaccination programmes (7), while in several countries, adolescent male vaccination has already been included into routine clinical practice (8). HPV has been proven to be an 'equal opportunity' pathogen affecting both sexes and adequate evidence supports the need to vaccinate adolescent boys and girls, before they become sexually active, in order to maximise the benefit of the highly effective HPV vaccines (9).

Greece was one of the first countries of the European Union, which introduced the HPV vaccination programme for girls aged 11-12 years into its national scheme. Currently, the National Immunisation Committee of Greece has excluded teenage boys from the national scheme (10), while the achieved rates among adolescent girls have been described to be unacceptably low (11). As it will be highlighted in the context of the 3rd Workshop on Paediatric Virology, which will be held on Saturday the 7th of October in Athens, Greece (12), 'if

Correspondence to: Professor Demetrios A. Spandidos, Department of Clinical Virology, School of Medicine, University of Crete, Heraklion 71003, Greece

E-mail: spandidos@spandidos.gr

Key words: human papillomavirus, Greece, 3rd Workshop on Paediatric Virology, Harald zur Hausen we really wish to eradicate these viral infections, we need to include boys into the vaccination programmes' (13). However, it is yet unclear what should the optimal clinical practice be in Greece, a country 'in the midst of a prolonged and deep economic recession that has already changed dramatically the lives of its citizens' (14). The question of whether boys should also be vaccinated is as important as the necessity to eradicate cervical cancer. In this direction, the vaccination of boys can indeed offer significant clinical, economic and ethical advantages $(15,16)$. Meanwhile, the increase in the currently low vaccination rates in females can represent a suitable target adding to the expected benefits that have been well described in the literature.

\section{References}

1. zur Hausen H: Papillomaviruses and cancer: From basic studies to clinical application. Nat Rev Cancer 2: 342-350, 2002.

2. Mammas IN and Spandidos DA: Paediatric Virology in the Hippocratic Corpus (Review). Exp Ther Med 12: 541-549, 2016.

3. Mammas IN, Sourvinos G and Spandidos DA: Human papilloma virus (HPV) infection in children and adolescents. Eur J Pediatr 168: 267-273, 2009.

4. World Health Organization: WHO position on HPV vaccines. Vaccine 27: 7236-7237, 2009.

5. Ekwunife OI, O'Mahony JF, Gerber Grote A, Mosch C, Paeck T and Lhachimi SK: Challenges in cost-effectiveness analysis modelling of HPV vaccines in low- and middle-income countries: A systematic review and practice recommendations. Pharmacoeconomics 35: 65-82, 2017.

6. Fesenfeld M, Hutubessy R and Jit M: Cost-effectiveness of human papillomavirus vaccination in low and middle income countries: A systematic review. Vaccine 31: 3786-3804, 2013.

7. Smith MA and Canfell K: Incremental benefits of male HPV vaccination: Accounting for inequality in population uptake. PLoS One 9: e101048, 2014.

8. Nanagas VC, Stolfi A, Nanagas MT, Eberhart GM and Alter SJ: Adolescent male human papillomavirus vaccination. Glob Pediatr Health 3: 1-9, 2016.

9. Gravitt PE: Human papillomavirus: The equal opportunity pathogen. J Infect Dis 215: 1014-1016, 2017.

10. National Immunisation Committee of Greece (Ministry of Health, Hellenic Republic): National Programme of Immunisations of children and adolescents 2017. http://www.isathens. gr/anakoinwseis/6906-ethniko-programma-emvoliasmwn-paidiwnefivwn-2017.html. Accessed July 31, 2017. 
11. Mammas IN, Theodoridou M, Koutsaftiki C, Bertsias G, Sourvinos $\mathrm{G}$ and Spandidos DA: Vaccination against human papillomavirus in relation to financial crisis: The 'Evaluation and Education of Greek Female Adolescents on Human Papillomaviruses' Prevention Strategies' ELEFTHERIA Study. J Pediatr Adolesc Gynecol 29: 362-366, 2016.

12. Mammas IN and Spandidos DA: Athens-based meeting to discuss the paediatric virology crossroad in October 2017. Acta Paediatr 106: 1536, 2017.
13. Mammas IN and Spandidos DA: Paediatric Virology as new educational initiative: An interview with Nobelist Professor of Virology Harald zur Hausen. Exp Ther Med 14: 3329-3331, 2017.

14. zur Hausen H: Support for Greece. Science 336: 978-979, 2012.

15. Mammas IN and Spandidos DA: Vaccination against human papillomavirus in childhood: The next rubella analogue? J BUON 17: 389-390, 2012.

16. zur Hausen H: HPV vaccines: What remains to be done? Interview by Lauren Constable. Expert Rev Vaccines 10: 1505-1507, 2011. 